\title{
Erratum: All in Your Mind? New-Onset Dysphagia in a Previously Healthy Adolescent Child
}

Jake Sequeira ${ }^{1}$ Douglas Willson ${ }^{1}$ Mark Marinello ${ }^{1}$

1 VCU Department of Pediatric Critical Care, Richmond, Virginia, United States

Address for correspondence Jake Sequeira, MD, VCU Department of Pediatric Critical Care, 1008 East Clay Street, Room B-009, Richmond,

J Pediatr Intensive Care 2020;9:e1. VA 23298, United States (e-mail: jake.sequeira@vcuhealth.org). included as the author in the above article in Journal of Pediatric Intensive Care, published online on December 5, 2019 (DOI: 10.1055/s-0039-3401008). The corrected author byline appears as given above. 